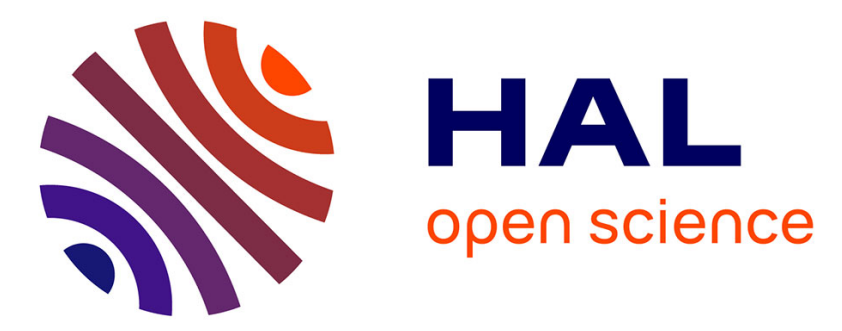

\title{
Spherical Radiative Transfer in C++ (SRTC++): A Parallel Monte Carlo Radiative Transfer Model for Titan
} Jason Barnes, Shannon Mackenzie, Eliot Young, Laura E Trouille, Sébastien Rodriguez, Thomas Cornet, Brian K Jackson, Máté Ádámkovics, Christophe Sotin, Jason Soderblom

\section{To cite this version:}

Jason Barnes, Shannon Mackenzie, Eliot Young, Laura E Trouille, Sébastien Rodriguez, et al.. Spherical Radiative Transfer in C++ (SRTC++): A Parallel Monte Carlo Radiative Transfer Model for Titan. The Astronomical Journal, 2018, 155 (6), pp.264. 10.3847/1538-3881/aac2db . insu-02780332

\section{HAL Id: insu-02780332 \\ https://hal-insu.archives-ouvertes.fr/insu-02780332}

Submitted on 4 Jun 2020

HAL is a multi-disciplinary open access archive for the deposit and dissemination of scientific research documents, whether they are published or not. The documents may come from teaching and research institutions in France or abroad, or from public or private research centers.
L'archive ouverte pluridisciplinaire HAL, est destinée au dépôt et à la diffusion de documents scientifiques de niveau recherche, publiés ou non, émanant des établissements d'enseignement et de recherche français ou étrangers, des laboratoires publics ou privés. 


\title{
Spherical Radiative Transfer in C++ (SRTC++): A Parallel Monte Carlo Radiative Transfer Model for Titan
}

\author{
Jason W. Barnes ${ }^{1}\left(\mathbb{D}\right.$, Shannon M. MacKenzie ${ }^{1}$, Eliot F. Young ${ }^{2}$, Laura E. Trouille ${ }^{3}$, Sèbastien Rodriguez ${ }^{4}$, Thomas Cornet ${ }^{4}$, \\ Brian K. Jackson ${ }^{5}$, , Máté Ádámkovics ${ }^{6}$, Christophe Sotin $^{7}$, and Jason M. Soderblom ${ }^{8}$ \\ ${ }^{1}$ Department of Physics; University of Idaho; Moscow, Idaho 83844-0903, USA; jwbarnes@uidaho.edu \\ ${ }^{2}$ Space Studies Department, Southwest Research Institute, Boulder, CO 80302, USA \\ ${ }^{3}$ Adler Planetarium, Chicago, IL 60605, USA \\ ${ }^{4}$ Institut de Physique du Globe de Paris (IPGP), CNRS-UMR 7154, Université Paris-Diderot, USPC, Paris, France \\ 5 Department of Physics, Boise State University, Boise, Idaho 83725-1570, USA \\ ${ }^{6}$ Physics and Astronomy Department, Clemson University, Clemson, South Carolina 29634, USA \\ 7 Jet Propulsion Laboratory, California Institute of Technology, Pasadena, CA 91109, USA \\ ${ }^{8}$ Earth, Atmospheric and Planetary Sciences, Massachusetts Institute of Technology, Cambridge, MA 02139, USA \\ Received 2017 October 27; revised 2018 April 24; accepted 2018 May 3; published 2018 June 4
}

\begin{abstract}
We present a new computer program, SRTC++, to solve spatial problems associated with explorations of Saturn's moon Titan. The program implements a three-dimensional structure well-suited to addressing shortcomings arising from plane-parallel radiative transfer approaches. SRTC++'s design uses parallel processing in an object-oriented, compiled computer language $(\mathrm{C}++)$ leading to a flexible and fast architecture. We validate $\mathrm{SRTC}++$ using analytical results, semianalytical radiative transfer expressions, and an existing Titan plane-parallel model. SRTC+ + complements existing approaches, addressing spatial problems like near-limb and near-terminator geometries, non-Lambertian surface phase functions (including specular reflections), and surface albedo nonuniformity.
\end{abstract}

Key words: planets and satellites: individual (Titan) - radiative transfer

\section{Introduction}

Titan's thick, extended, hazy atmosphere elicits complex interactions of incoming photons with aerosols, gases, and the surface. Scattering by haze aerosol particles smears visibility of Titan's surface, particularly at short wavelengths where haze particles have large extinctions (Smith et al. 1981; Richardson et al. 2004; Porco et al. 2005; Tomasko \& West 2010; Maltagliati et al. 2015a). Gaseous methane, nitrogen, and carbon monoxide in Titan's atmosphere absorb light at most near-infrared wavelengths, permitting transmission from the surface only within distinct spectral windows (Griffith 1993; Smith et al. 1996; Barnes et al. 2007; Vixie et al. 2012). And Titan's heterogeneous surface (Griffith 1993; Barnes et al. 2005) exhibits spectral units with varying reflectance phase functions, including specular reflections off the smooth liquid lakes and seas (Stephan et al. 2010; Soderblom et al. 2012; Barnes et al. 2013). We illustrate some of these effects in Figure 1.

Given the complex geometry and processes involved, inversion of reflectance spectra to independently ascertain surface and atmospheric parameters has proven challenging. Currently working models for Titan typically assume a planeparallel one-dimensional (1D) atmosphere (e.g., McKay et al. 1989; Rannou et al. 2003; Rodriguez et al. 2006; Griffith et al. 2012a; Maltagliati et al. 2015b; but see also Xu et al. 2013, which uses a spherical-shell assumption instead of plane-parallel). And they do so for good reasons. Quality 1D algorithms exist and have been thoroughly tested, and 1D codes' approximations allow for fast radiative transfer calculations, thus enabling their practical use for spectral modeling (Young et al. 2002; Hirtzig et al. 2013) and the inversion of surface albedo (Coustenis et al. 1995; Griffith et al. 2012a; Solomonidou et al. 2014; Maltagliati et al. 2015b; Solomonidou et al. 2016; Ádámkovics et al. 2016).
Some subsets of Titan problems do not lend themselves well to being solved using 1D plane-parallel codes, however. Titan's extended atmosphere leads to a dramatic falloff in the accuracy of the 1D plane-parallel approximation beyond incidence and emission angles of $\sim 60^{\circ}$ (with significant deviations at geometries as low as $\sim 35^{\circ}-40^{\circ}$ incidence for an extended atmosphere like Titan's). Hence, observations near Titan's limb, or near its poles, cannot be modeled using existing algorithms without complex modifications. One-dimensional codes by their nature do not model spatial problems like the adjacency effect, simulations of spatial resolution capabilities, indirect illumination beyond the terminator, and non-Lambertian surface phase functions.

To solve this spatial class of Titan radiative transfer problems, we have written a new radiative transfer computer program. The new algorithm uses a Monte Carlo approach in conjunction with fully spherical atmospheric geometry so that it can be used at extreme geometries and to solve spatial problems. Although the new code tracks photons in parallel, its speed still leaves much to be desired relative to conventional 1D plane-parallel approaches (e.g., Griffith et al. 2012b; Solomonidou et al. 2014). Hence, our new approach is designed to complement, and not to supplant, the current generation of radiative transfer solvers.

Although our work resembles existing astrophysical Monte Carlo radiative transfer solvers, like Hyperion (Robitaille 2011), SUNRISE (Jonsson 2006), MC3D (Wolf 2003), and SKIRT (Baes et al. 2003) in many aspects, our strengths lie in our design based around planetary remote sensing problems. Although our approach is general and could in principle be adapted for other purposes, we assume a central spherical solid body and allow for multiple observation viewpoints as might be seen from spacecraft. Together, these design choices allow both easier problem setup and more rapid and efficient computation of the results. 

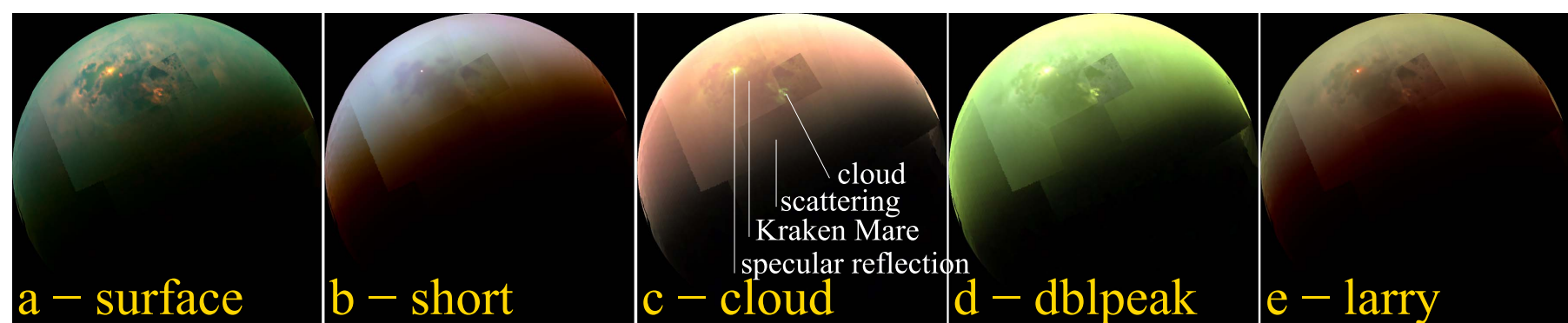

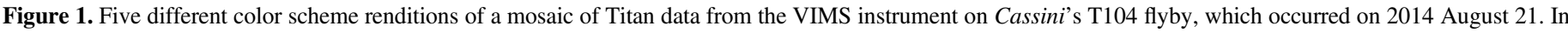

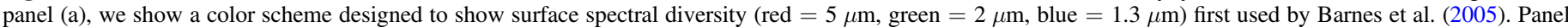

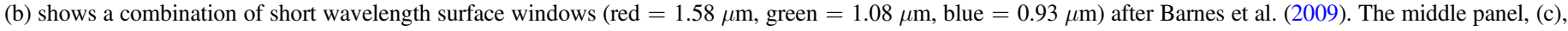

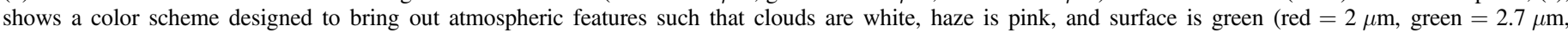

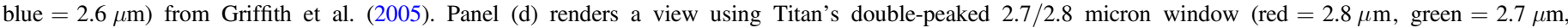
blue $=2.0 \mu \mathrm{m})$. Panel (e) at right shows the surface color scheme developed by Soderblom et al. $(2007)(\mathrm{red}=2 \mu \mathrm{m}$, green $=1.58 \mu \mathrm{m}, \mathrm{blue}=1.28 \mu \mathrm{m})$.

We call the new code SRTC++ for "Spherical Radiative Transfer in C ++ ". We describe the nuts and bolts of the code itself, including the treatment of atmospheric structure, the generation of input photons, the photons' traverse through that atmosphere, and our detector implementation in Section 2. We then validate our results by comparing to the results of planeparallel radiative transfer models in Section 3. Benchmarking discussions proceed in Section 4. Finally, we conclude with discussion of future application of SRTC++ in Section 5.

\section{Computation}

Spherical Radiative Transfer in $\mathrm{C}++(\mathrm{SRTC}++)$ is based on an earlier algorithm, SRTC, which was written in Interactive Data Language (IDL) by two of us (EFY and LET) but never published. We make no assumptions about atmospheric structure or scattering qualities, but rather virtually throw photons toward the target and probabilistically calculate their behavior. We first calculate where the photon experiences a scattering event, which we determine randomly first in optical depth space and later in physical space. At each of these randomly determined scattering events, we update the detector to record the visible response corresponding to the scatter. Then we probabilistically calculate a new direction for the photon post-scatter from the scattering phase function.

Because we directly simulate the experience actual photons might have interacting with the target planet, the approach is simple, straightforward, and broadly applicable. There are no Markov Chains, no scattering order assumptions, no assumptions of homogeneity or of a plane-parallel atmosphere. However, because it does not make any of these assumptions that could speed it up, the algorithm is slow. We designed $\mathrm{SRTC}++$ to significantly improve on the original SRTC through translation into a fast, compiled language $(\mathrm{C}++)$, and by implementing SRTC++ in parallel from the ground up, potentially to speedup computation by a factor of the number of machine CPU cores.

\subsection{Component Data Structures and Methods}

Before diving in to the primary code execution loop, we first describe the general setup requirements and class structures that SRTC++ uses.

\subsubsection{Atmosphere}

In SRTC++, an atmosphere is a list of atmospheric layers. The layers start with the lowest layer, a type of "atmospheric" layer that corresponds to the surface. Atmospheric layers, or atmolayers, know their own vertical depth, scale height, and name, and contain pointers to the layers above and below them. The last layer of atmosphere always points to the static "Space ()" layer. SRTC++ integrates the opacity through each separate layer individually. The user can therefore decide to implement multiple homogeneous layers with different properties or a single giant layer with internally varying properties. Note that the former may run faster for numerical integrations in cases where properties exhibit discontinuities-numerical integration typically slows down severely when integrating discontinuous functions.

Each atmolayer contains a vector of atmospheric zones, or atmozones. Atmozones know their haze scattering and gas absorption normal optical depth properties (separately), along with single-scattering albedo and scattering phase function properties. A simple atmosphere would typically contain just a single atmospheric zone, but this structure allows for arbitrarily complex surface or atmospheric variations with latitude, longitude, and altitude. In particular, albedos including surface albedo can also be set with a raster image jpeg to allow for intricate and complex structures (see Figure 2).

\subsubsection{Photon Generation}

SRTC++ starts with initial incident light from a photon generator. The photon generator takes a photon identification number (photon ID) from which it infers the initial state of the photon. Photons have knowledge of their $x, y$, $z$ position, and their $V_{x}, V_{y}, V_{z}$ direction along with their amplitude $^{9}$ (initially set to 1.0) and wavelength. Our simple "square" photon generator returns a raster of photons in $y, z$ space all initially pointed in the $-x$ direction and located outside Titan's atmosphere at $x=+4200 \mathrm{~km}$.

The photon generator is an abstract class, and therefore one from which users can build as a basis for new photon generators that work differently.

There are two essential aspects of the photon generator. (1) At no time are all of the photons stored in memory, thereby reducing the program's memory footprint and allowing an arbitrarily large number of input photons not limited by memory size. (2) Because each photon is produced from a

\footnotetext{
Of course actual, physical photons do not have varying amplitudes. Our treatment of photons by referring to their "amplitude" is equivalent to what other Monte Carlo models might call a "photon packet" and corresponds to the aggregate behavior of a large number of photons and not to an actual single individual photon.
} 


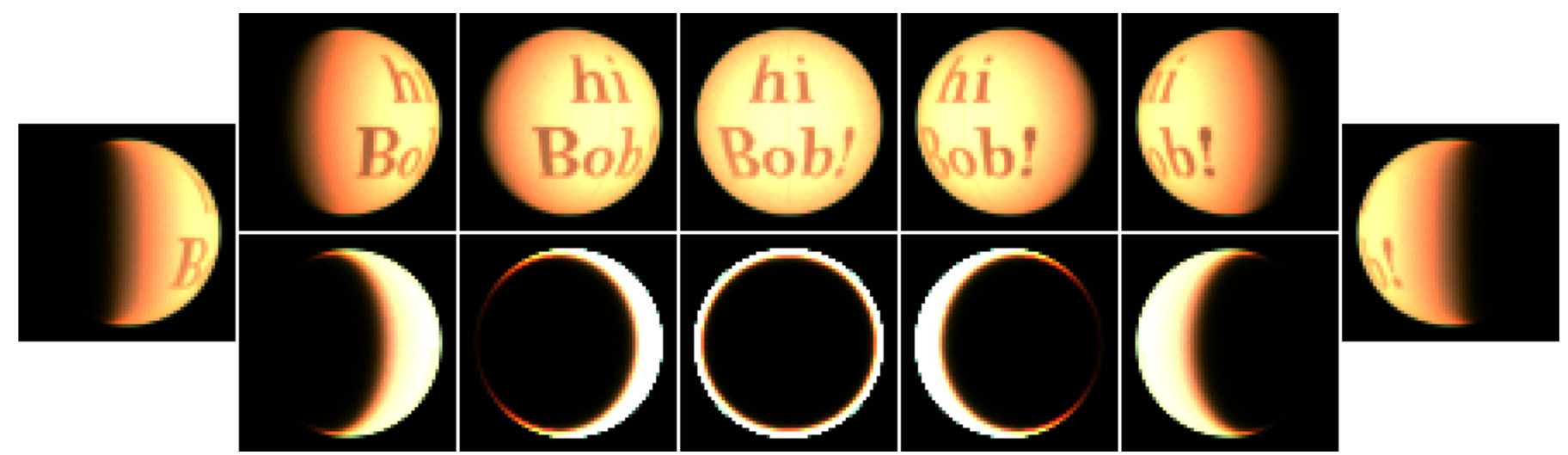

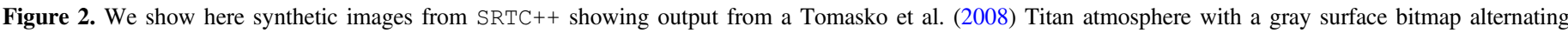

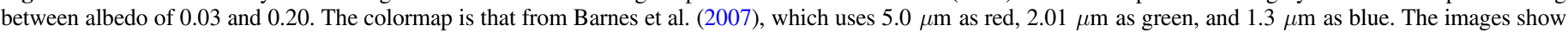

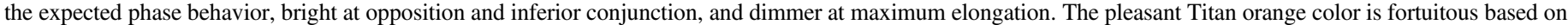

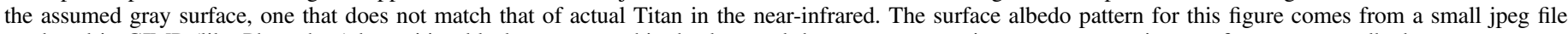
produced in GIMP (like Photoshop) by writing black text on a white background, but SRTC++ can import any raster image of atmozone albedos.

single initial photon ID number, each photon is entirely independent of every other photon, not relying on them for any previous knowledge of position or wavelength. Aspect (1) allows for ambitious models with high photon counts and therefore high signal-to-noise ratios (S/Ns), and aspect (2) allows each photon to proceed in parallel disregarding when or on what processor it is running.

The simplest built-in photon generator, photongenerator_square, implements a regularly spaced raster of photons with a given side length in kilometers, number of rows and columns $n$, and list of $w$ different wavelengths. Every photon generator knows its size-the total number of photons that it expects to generate $\left(n^{2} w\right)$. The primary loop then starts a long integer iterator $i$ at $i=0$ and runs through to the photon generator size, passing $i$, the photon ID number, to the generatephoton ( ) method at the beginning of the loop. This method then determines the wavelength to be the entry in the input list corresponding to the integer value of $i / n^{2}$. Then, using the remainder $r \equiv i \% n^{2}$, the row number comes from the integer value of $r / n$ and the column from the remainder $r \% n$. From the row and column number, we determine the initial $y$ and $z$ coordinate locations for the photon. The photons' initial directions are all the same: $-x$ for photongenerator square (though the initial direction could be assigned differently in an updated photon generator, to come from a point source like the Sun for instance instead of being parallel). The output of generatephoton() is an instance of the photon class that knows its own three-dimensional (3D) vector position, its direction, and its "amplitude" (a measure of what fraction of the virtual photon has been previously absorbedwhich of course does not really happen for a single photon, so our photon class might more accurately be thought of as a bundle of photons).

This backwards approach certainly takes a few extra CPU cycles than a forward calculation of $y, z$, and wavelength in a triple loop. But it reduces memory accesses and storage and allows threads to run independently and for photon ID numbers to run unordered and randomly as assigned by the parallelization.

\subsubsection{Detectors}

In SRTC++, detectors are implemented as an abstract class-hence, any different kind of detector could be created as might be helpful to solve any particular future problem. To start, we created a simple detector called colorCCD. A ColorCCD knows its position and the direction in which it is pointed. It has a virtual two-dimensional (2D) CCD array with multiple planes to allow for different wavelengths that are calculated simultaneously.

Distinct from existing 1D codes, which typically have as their output a spectrum that results from a particular atmosphere and input geometry, ColorCCD records images (like those in Figure 2). Pixels in the image correspond to different locations on the target planet (or off its limb) and therefore to different local incidence, emission, and azimuthal angles. Because the SRTC++ treatment is $3 \mathrm{D}$, conditions in one area may affect nearby pixels in the ColorCCD.

$\mathrm{SRTC}++$ takes a vector of detectors as input. It follows that it can use the same radiative transfer calculation to update any number of detectors during a single run. Figure 2, for instance, was created in a single SRTC++ configuration that used 36 different detectors arrayed around Titan's equator every $10^{\circ}$.

For testing purposes (see Section 3), we also introduce a more complex detector: the elephant detector. It never forgets photon histories. Rather than store the full history of a billion photons, though, elephant detectors have separate colorCCDs for each possible photon scatter path topology. We denote each photon history using a " 0 " for surface scatters and a " 1 " for atmospheric scatters. We end up with one ColorCCD for just surface scatters (_0) and one for just one atmospheric scatter (_1). We store each scenario separately for double scatters: surface then atmosphere(_01), atmosphere then surface (_10), and photons that scatter twice off the atmosphere (_11). Although elephant formally tracks surface-then-surface (_00) scatters, too, in practice there should not ever be any of these for spherical planets (though there would be if SRTC++ modeled topography-future work). In general, there should be $2^{n}$ different ColorCCDs per scattering order $n$ (e.g., for $n=3$ : $000,001,010,011,100$, 101, 110, and 111). Elephants allow the user to set the maximum scattering order of which to keep track.

\subsection{Main Program Loop}

Independent parallel execution threads each start with a photon ID. From this ID, the photon generator determines the 
initial position, direction, and amplitude for the photon in question and sends it into the main program loop.

The main loop is as follows:

(1) plots out the photon's path forward and backward from its present location, turning that path into a $1 \mathrm{D}$ photon traverse through the atmosphere (details in Section 2.2.1);

(2) generates a random excursion for this photon in scattering optical depth space $\tau_{\text {scat }}$ (details in Section 2.2.2);

(3) translates that random $\tau_{\text {scat }}$ into a physical location for scattering along the photon traverse (details in Section 2.2.3)-allowed types of scatter are surface, atmosphere, or space (the latter indicates that the photon has escaped the atmosphere, in which case its amplitude is updated to 0.0 );

(4) updates the detectors (details in Section 2.1.3) based on this scatter; and

(5) attenuates the photon's amplitude by the single-scattering albedo at the scatter location, and updates the photon's direction based on the local scattering phase function (details in Section 2.2.4).

The resulting new photon position, direction, and amplitude then inform the next iteration of the loop. Each thread continues calculating its photon's path through scattering events until that photon either flies off into space or its amplitude becomes identically 0.0 (as would happen if the albedo were zero either somewhere in the atmosphere or at the surface), at which time the loop terminates and the thread asks for a new photon ID to calculate.

We describe the details of each of these steps in the following sections.

\subsubsection{Photon Traverse}

A photon traverse class instantiation translates the photon's 3D position and direction into a $1 \mathrm{D}$ path. The path is centered on the photon's present location, and we parameterize the distance from that location as $d$ such that negative values are behind the photon and positive values are ahead of it. Armed with this coordinate collapse, we then can determine the atmospheric conditions at any point along the photon's traverse.

Specifically, the photon traverse looks up which atmolayer and atmozone corresponds to each value for $d$. The most important conditions that we need are the volume extinctions $\kappa(\lambda)$ at each point. We then numerically integrate that extinction (using qromb from Press et al. 1992) along the photon's traverse to calculate the cumulative $\tau$ between the photon's present position and each position $d$. While the original IDL code on which SRTC++ builds uses an analytic approximation to the Chapman Integral (Smith \& Smith 1972), we instead evaluate the integrated optical depth numerically. While the numerical approach slows program execution, Checlair et al. (2016) shows that the compromises inherent to the (Smith \& Smith 1972) approximation in puffy atmospheres like Titan's result in $\gtrsim 10 \%$ systematic errors. It follows that we find the slow-

but-accurate numerical calculation preferable.

Figure 3 shows an example of how we calculate such an integral. The red line represents the photon traverse next to Titan (a near-grazing impact parameter starting from deep space for this particular photon), and the plot above the red line

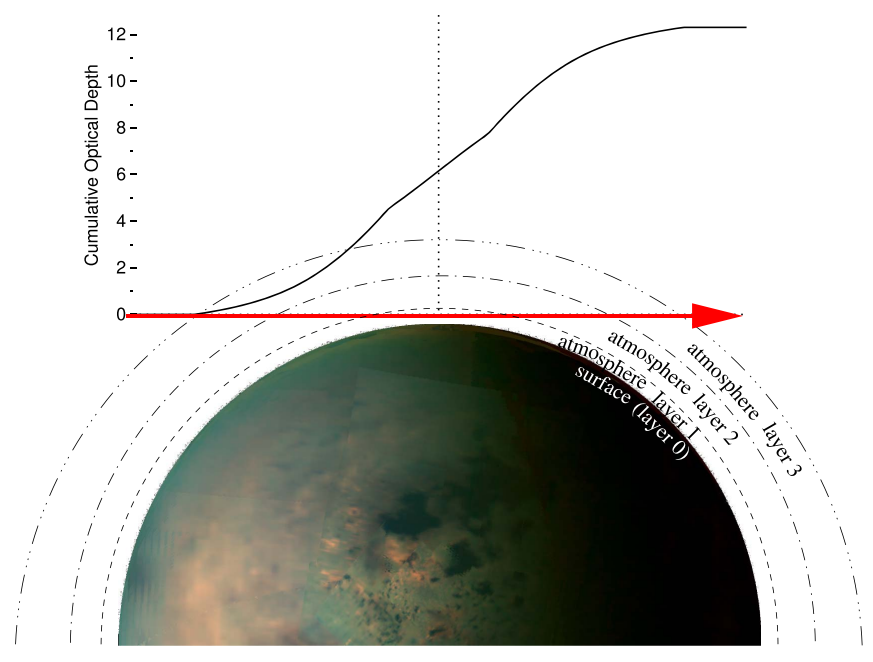

Figure 3. This figure shows a stylized representation of an example photon traverse through Titan's atmosphere. The plot shows the cumulative optical depth as a function of distance along a photon traverse indicated by the red ray. SRTC++ uses this type of cumulative optical depth function within a photon traverse to both calculate net atmospheric opacity and to ascertain the location in physical space at which a scattering event occurs, translating from optical depth space. We do that by generating a random photon optical depth $\tau_{\text {random }}$ at which the next scatter occurs from Equation (1) and then tracing that $\tau_{\text {random }}$ to its corresponding location along the traverse in this plot. This particular photon's photon traverse heads in from deep space, encounters Titan's outer atmosphere, and then continues down before reaching a closest approach in the troposphere but above the surface. It then heads back out into space. The breaks in slope occur at the interfaces between atmospheric layers as specified in the Tomasko et al. (2008) atmospheric model.

shows the integrated haze optical depth at each point along the traverse. We take advantage of this knowledge of the integrated optical depth along the path for Steps 3 and 5.

\subsubsection{Generating Random Optical Depths}

We need to calculate a location where a photon might scatter in optical depth $\tau_{\text {scat }}$ space. The probability of it scattering at low $\tau$ is much greater than that at higher $\tau$. (By higher $\tau$, more of the photons have been already scattered out of the beam.) Achieving this aim requires the ability to generate random numbers between 0.0 and $+\infty$ with an exponentially decaying distribution as $e^{-\tau}$.

To do so, we follow Press et al. (2007). First, we generate a random number $f_{\text {random }}$ between 0.0 and 1.0 using a modified version of the Numerical Recipes routine Ranq1 Press et al. (2007). The modifications allow for parallelization by creating different instances of the random number generator (seeded with different initial values) for each thread so that they do not interfere with one another. To get the appropriate random $\tau_{\text {random }}$ we then set

$$
\tau_{\text {random }}=-\ln f_{\text {random }} .
$$

\subsubsection{Determining the Scattering Location}

Having generated a random scatter location along $d$ in $\tau$ space (at $\tau_{\text {random}}$ ), we then need to invert the $\tau$ versus $d$ plot in Figure 3 to determine the value of $d$ corresponding to $\tau_{\text {random }}$. From $d$, we then infer the precise location in 3D space where the scatter will occur. SRTC++ determines the appropriate $d_{\text {random }}$ for the random input $\tau_{\text {random }}$ numerically using the Ridders' Method zero-finding routine zriddr from Press et al. (1992). 


\subsubsection{Updating the Detectors}

After the scattering location has been determined, but before we determine the result of the scatter itself (i.e., the photon's direction and amplitude moving on from this scatter), we update the detector to reflect the response that it would see from this scattering event (Yusef-Zadeh et al. 1984; Dupree \& Fraley 2012; Jonsson 2006). This step represents the critical insight of our Monte Carlo approach: instead of a complete Monte Carlo simulation of light scattering, in which only a very few photons would find their way to the detector, we instead accumulate contributions of each photon at the detector as generated by each scattering event. And then we throw away the the Monte Carlo photons after they leave Titan's atmosphere to avoid double-counting. This approach has come to be known as the "peeling-off" technique.

So, let us restate this critical insight in another way. We use the Monte Carlo photons pinging around through the atmosphere to determine amplitudes, locations, and geometries for scattering. But we never record the results of the primary photon itself at any detector. Every photon eventually makes its way out of the atmosphere, at which time we ignore it and move on to the next photon to be calculated. Instead at the instance of each scattering, and separately for each detector, we calculate the explicit contribution that the particular scattering in question would have at those detectors. It follows that the detection step is not Monte Carlo but instead is determinitive allowing for efficient use of computation power.

The detector is invoked with a call that passes a photon-the photon with its location as the location of the scatter but with the direction of the photon prior to this particular scattering event. The detector then calculates the relative scattering angle $\phi_{d}$ between its position and the photon, recording the effective charge $c$ received at the detector based on the photon's initial amplitude $A_{n}$, the scattering phase function $p\left(\phi_{d}\right)$, the optical depth between the scatter and the detector $\tau_{\text {outbound }}$, and the single-scattering albedo of the atmosphere at the point of scatter $\omega$ (note that we treat scattering and gaseous absorption together such that $\omega$ represents both components)

$$
c(x, y, \lambda)+=A_{n} \omega p\left(\phi_{d}\right) e^{-\tau_{\text {outbound }}} .
$$

We do this increment separately for each of the detectors in the vector set up for this particular run.

Then, at the end of the entire SRTC ++ run when all of the photons are finished, we calibrate the detector into $I / F$ by

$$
\frac{I}{F}=\frac{1}{\pi} \sigma \Upsilon^{2} c(x, y, \lambda)
$$

where $\sigma$ represents the number of photons incident per square kilometer according to the photon generator and $\Upsilon$ is the ColorCCD sampling in kilometers per pixel.

\subsubsection{Calculating a New Direction after Scattering}

Lastly, for each photon, SRTC++ executes the scattering in a Monte Carlo fashion. We decrease the photon's amplitude according to the single-scattering albedo of the atmosphere. SRTC++ calculates the new direction by (1) using the phase function to calculate a cumulative distribution function (fraction of scattered light scattered by an angle less than or equal to the ordinate), (2) inverting that cumulative distribution function into angle as a function of fraction, and (3) interpolating the inverse cumulative distribution function to find the right new angle. Step 3 involves generating a random number in the usual uniform $0.0-1.0$ variate fashion, finding where that value would fall in the inverse cumulative distribution function, and then interpolating to find the actual scattering angle implied. Because generating and inverting the cumulative distribution in Steps 1 and 2 depend only on the phase function, we calculate the inverse cumulative distribution functions prior to entering the primary code loop and then all threads share access to the results. Importantly, this approach allows for use of arbitrary phase functions and/or different phase functions in different portions of the atmosphere-we have no requirement that a phase function be decomposable into Henyey-Greenstein or polynomial expansions.

Once we have decided upon a scattering angle, we generate a random azimuth between 0 and $2 \pi$. Then comes a kind of tricky part. The scattering angle and azimuth are valid for a coordinate system oriented along the direction of travel of the photon prior to the scattering event. So we transform the initial $x, y, z$-basis direction vector into one in this new reference frame where the photon travels in $+x$ prior to the scatter. Next, we use the new random scattering angle and azimuth to determine the photon's outgoing direction after the scatter, but still in the reference frame based on the direction prior to the scatter. Lastly, we transform this outgoing vector back into $x, y$, $z$-space and send this post-scatter photon back around the loop for another round.

\section{Validation}

Any new radiative transfer scheme can only start to be trusted after it faithfully reproduces known endmember test cases. To that end, we use a series of validation test cases to verify that $\mathrm{SRTC}++$ reproduces known cases at a satisfactory level. We use analytic and existing numerical plane-parallel models as our primary comparitors due to their accuracy at high optical depths. (Optically thin and single-scattering approximations to spherical atmospheres (i.e., Rages \& Pollack 1983; Squyres et al. 1985) might also be relevant in future contexts.)

\subsection{Lambertian Surface and Thick Atmosphere}

The first, simplest check that we run verifies that bare surfaces and infinitely deep atmospheres modeled with SRTC+ + yield comparable answers to analytical results.

For the surface case, we use an albedo equals 1.0 Lambert sphere in combination with an extremely tenuous $1 \times 10^{-6}$ optical depth atmosphere. We verified that this setup produces an average $I / F$ of $2 / 3$ when integrated across the entire disk from $0^{\circ}$ phase.

We next compare SRTC++'s results for a high-optical-depth atmosphere $30-\mathrm{km}$ thick (here assumed to be $\tau_{\text {total }}=5.0$ with uniform extinction for testing purposes-higher $\tau$ takes longer to calculate with diminishing returns) to the theoretical results for an plane-parallel, optically thick atmosphere as calculated by Chandrasekhar (1950). Chandrasekhar (1950) shows that

$$
I(0, \mu)=\frac{1}{4} \omega F \frac{\mu_{0}}{\mu+\mu_{0}} H(\mu) H\left(\mu_{0}\right)
$$

where $I$ is the incident intensity, $F$ is the resulting flux field, $\omega$ is the single-scattering albedo, and $\mu$ and $\mu_{0}$ are the cosines of the angles of the observer and the source (the Sun) with respect to the zenith direction (Chandrasekhar (1950), Equation (26)-(118). $H()$ is Chandrasekhar's H-function. 


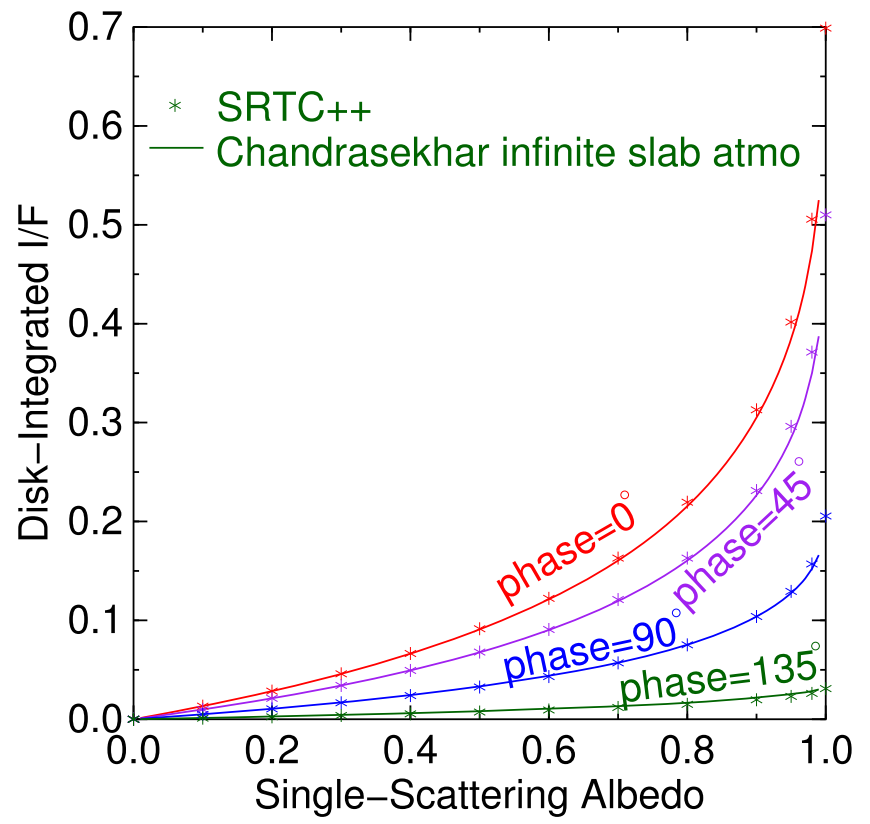

Figure 4. We show here a comparison between disk-integrated $I / F$ values for an optically thick atmosphere viewed at $0^{\circ}, 45^{\circ}, 90^{\circ}$, and $135^{\circ}$ phase. We assume an isotropic atmospheric scattering phase function. The results calculated by SRTC $++($ asterisks $)$ and by numerical integration of Chandrasekhar's slab equation (solid lines, from Equation (6)) substantially agree with one another for this simple case.

We rewrite Equation (4) to switch out $\mu$ and $\mu_{0}$ in favor of $\alpha$ s instead, which equate to the number of traversed atmospheres in the relevant geometry from a plane-parallel atmosphere. So

$$
\alpha=\frac{1}{\mu}=\frac{1}{\cos e}
$$

and

$$
\frac{I(0, \alpha)}{F}=\frac{1}{4} \omega \frac{\alpha}{\alpha+\alpha_{0}} H(\alpha) H\left(\alpha_{\odot}\right) .
$$

We show the resulting comparison of numerical integrations of Equation (6) over the full disk, as compared to results from SRTC++, in Figure 4 (see also tabulations of brightness distribution across the disk in this case from Table 3 in Dlugach \& Yanovitskij 1974). We assume an isotropically scattering atmospheric phase function in both cases for simplicity (we use more complex atmospheric phase functions in later tests outlined below). Figure 4 shows four different curves in different colors - one for the spherical planet as viewed at four different phase angles $\left(0^{\circ}, 45^{\circ}, 90^{\circ}\right.$, and $\left.135^{\circ}\right)$. The SRTC ++ result closely tracks the theoretical result of the Chandrasekhar slab all the way up to just below a single-scattering albedo of 1.0 where the theoretical value no longer applies. It follows that SRTC++ works for this simple phase function in a multiplescattering regime.

\subsection{Atmosphere and Surface}

For the next verification step, we compare SRTC++ results to those of a more general semianalytic model. We use a model derived by one of us (BKJ) from Thomas \& Stamnes (2002) and initially used in Vixie et al. (2015). The model separately calculates $I / F$ contributions from different scattering histories involving at most one atmospheric scatter. Specifically, we
Table 1

Atmospheric Parameters Assumed for the Orange-rind Atmosphere Validation Described in Section 3.2

\begin{tabular}{lcc}
\hline \hline Wavelength & Optical Depth & Single-scattering Albedo \\
\hline $0.93 \mu \mathrm{m}$ & $\tau_{0.93}=3.2$ & $\omega_{0.93}=1.00$ \\
$1.08 \mu \mathrm{m}$ & $\tau_{1.08}=2.5$ & $\omega_{1.08}=0.99$ \\
$1.28 \mu \mathrm{m}$ & $\tau_{1.28}=2.1$ & $\omega_{1.28}=0.98$ \\
$1.58 \mu \mathrm{m}$ & $\tau_{1.58}=1.45$ & $\omega_{1.58}=0.96$ \\
$2.0 \mu \mathrm{m}$ & $\tau_{2.0}=1.02$ & $\omega_{2.0}=0.77$ \\
$2.68 \mu \mathrm{m}$ & $\tau_{2.68}=0.8$ & $\omega_{2.68}=0.507$ \\
$2.78 \mu \mathrm{m}$ & $\tau_{2.78}=0.8$ & $\omega_{2.78}=0.463$ \\
$5.0 \mu \mathrm{m}$ & $\tau_{5.0}=0.3$ & $\omega_{5.0}=0.4998$ \\
\hline
\end{tabular}

look at the terms that correspond to: (1) photons that make it through the atmosphere, scatter off the surface, and make it all the way out unscathed; (2) photons that scatter off the atmosphere once and then back to the detector; (3) photons that scatter first off the atmosphere, and then off the surface, before being detected; and (4) photons that scatter off the surface first, then scatter off the atmosphere on their way out. Using elephant detectors in SRTC ++ , then, we can compare the Monte Carlo result directly to the semianalytical result for each scattering history individually.

In this comparison, we assume a uniform-extinction, 30-kmthick atmosphere with haze that scatters according to the Tomasko et al. (2008) phase function below $80 \mathrm{~km}$. For purposes of the test, we assume optical depths $\tau$ and singlescattering albedos $\omega$ as shown in Table 1. These values are consistent with both Huygens results (Tomasko et al. 2005) and Cassini measurements of atmospheric transmission within Titan's spectral windows (Barnes et al. 2013; Hayne et al. 2014; Maltagliati et al. 2015a). As we intend to test our model and not simulate actual Titan in this particular instance, we set the surface albedo $A=1.0$ and we ignore gaseous absorption.

On the SRTC++ side, this model run uses 22,606,068 photons, illuminating the full disk of Titan. We install 18 different detectors, each elephants in this case, spread out in phase angle from the Sun from $0^{\circ}$ to $170^{\circ}$ in $10^{\circ}$ increments. This run took overnight to complete.

\subsection{One Scatter at Surface (__ 0$)$}

The first term corresponds to scatter only from the surface,

$$
\frac{I_{-} 0}{F}=\pi A \Phi_{S}(i, e, \phi) e^{-\tau(\alpha(i)+\alpha(e))}
$$

where $i$ is the incidence angle, $e$ is the emission angle, and $\phi$ is the phase angle. $I_{-}{ }_{0}$ corresponds to the intensity at the detector for light that only reflects off the surface one time and that is not extincted by the atmosphere on the way in or the way out. We convert this to the more easily interpreted $I / F$ by dividing by the solar flux $F$. $A$ is the surface albedo. $\Phi_{s}(i, e, \phi)$ refers to the surface phase function (hence the $s$ ), which could potentially be a function of the incidence, emission, and phase angles. Finally, $\tau$ is the one-way atmospheric optical depth at normal geometry (i.e., looking straight down or up through the atmosphere), and the $\alpha$ functions of incidence $(i)$ and emission (e) correspond to the number of atmospheres traversed by photons in this geometry under the plane-parallel approximation-so $\alpha(i)=\frac{1}{\cos i}$. 


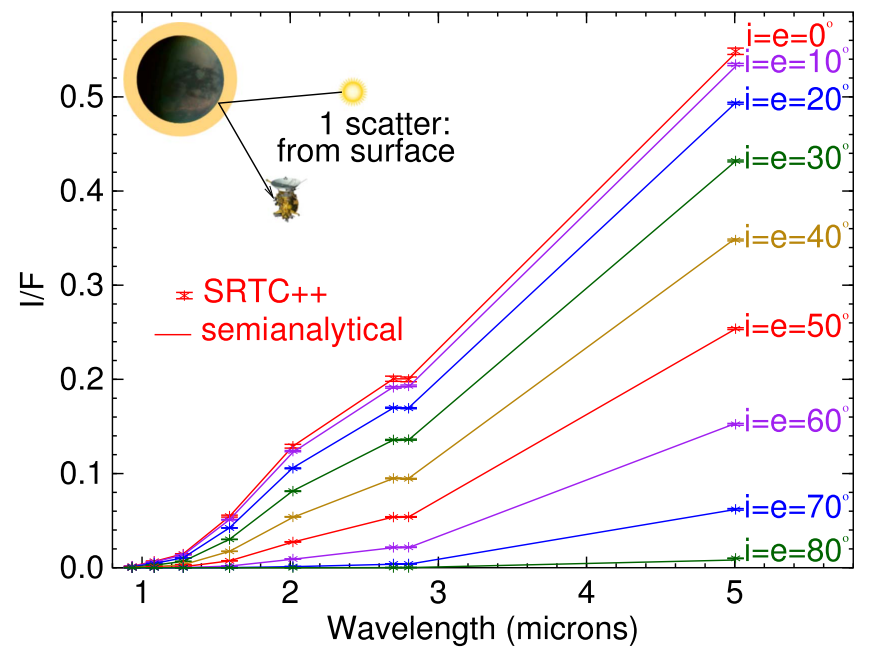

Figure 5. This figure is the first of four plots comparing SRTC++ to a semianalytical model (described in Section 3.2) at different scattering orders. For each of these four plots, we assume a $30-\mathrm{km}$ deep orange-rind atmosphere with the scattering phase function of Tomasko et al. (2008) for below $80 \mathrm{~km}$ (to test SRTC++'s use of complex phase functions). The optical depth $\tau$ and single-scattering albedo $\omega$ were chosen to be representative of Titan within each window. This particular plot shows $I / F$ as a function of wavelength within Titan's near-infrared atmospheric windows for light that only scatters off the surface and not from the atmosphere. Both approaches arrive at the same answers for this (relatively simple) case.

We show the results for the 1-scatter from surface case in Figure 5. In this plot and the other comparisons to the semianalytical model, we generate our error bars empirically. For each geometry (i.e., $i=e=20^{\circ}$ ), we collect all of the pixels in the COlorCCD that match the incidence and emission to within a given tolerance (here we use $5^{\circ}$ ). We assign the asterisk SRTC++ value in Figure 5 to the average of the pixels in that collection, and the error to their standard deviation. Because that collection includes a wide diversity of phase angles between $\phi=0^{\circ}$ and $\phi=i+e$, this approach overestimates the errors. But because we compare the same set of pixels in both the SRTC++ and semianalytical cases, the validation comparison remains valid. SRTC++ and the semianalytical approach agree within very tight tolerancesnot entirely unexpected as this case is particularly simple compared to multiple-scattering cases.

\subsection{One Scatter in the Atmosphere (_1)}

In the semianalytical model, light that scatters only once in the atmosphere has an intensity of

$$
\frac{I_{-1}}{F}=\pi \omega p(i, e, \phi) \frac{\alpha(e)}{\alpha(i)+\alpha(e)}\left(1-e^{-\tau(\alpha(i)+\alpha(e))}\right)
$$

where new parameter $\omega$ corresponds to the atmospheric singlescattering albedo and new function $p$ to the atmospheric scattering phase function, which potentially depends on the incidence, emission, and phase. We compare the results of this equation and that of SRTC++ in Figure 6.

The resulting plots show more complicated behavior than those for the surface-only case. At longer wavelengths (i.e., at $5 \mu \mathrm{m})$, the expected $I / F$ increases monotonically with viewing angle (we assume that the incidence angle equals the emission angle so as not to end up with $9^{2}$ different lines in the plot) as the higher path length increases the slant optical depth. For shorter wavelengths, though, like at $0.93 \mu \mathrm{m}$, the situation gets

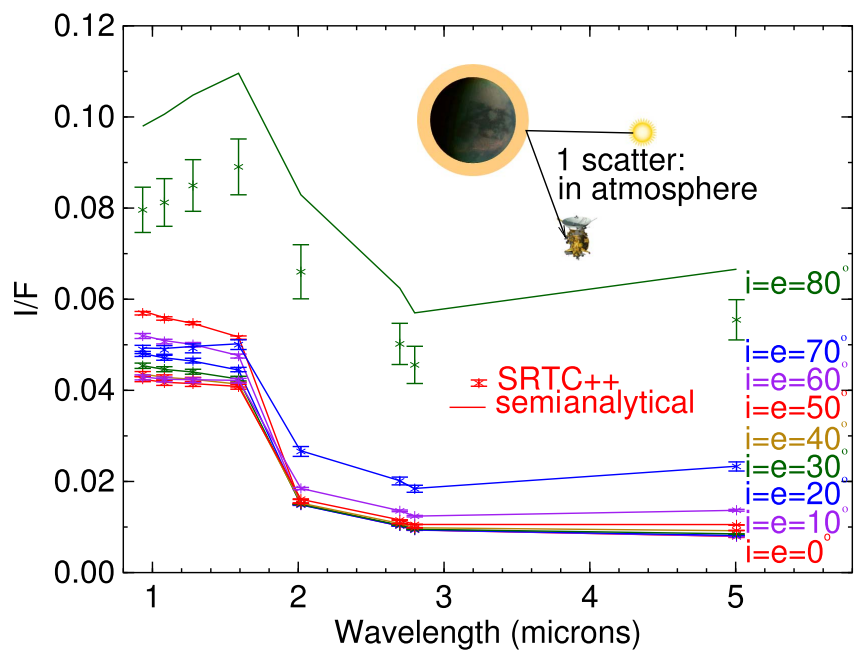

Figure 6. We again compare $\mathrm{SRTC}++$ results to semianalytical results, in this case for photons that only scatter one time within the atmosphere. The solid line plots the result of Equation (8) while the asterisks with error bars show SRTC++'s answer. The analytical model assumes plane-parallel geometry, and hence is well outside its range of validity by the time $i=e=80^{\circ}$.

more complicated. The $i=e=0^{\circ}$ case shows up as the dimmest. But instead of a monotonic increase, the $0.93 \mu \mathrm{m} I / F$ peaks at $i=e=20^{\circ}$ before decreasing and later increasing again toward very high incidence and emission. The more highly forward-scattering phase function at shorter wavelengths causes the disparity. Note the interesting case where the two blue curves, $i=e=20^{\circ}$ and $i=e=70^{\circ}$ both have the same $I / F$ at $0.93 \mu \mathrm{m}$ but then diverge as they head toward longer wavelengths and lower optical depths. This effect is a bit unintuitive, but shows up in both models as SRTC++ and the semianalytic model track each other well up to $i=e=70^{\circ}$.

At $i=e=80^{\circ}$, though, the two models disagree. Presumably, this discrepancy results from applying the semianalytic model, which assumes plane-parallel geometry, beyond the point where it produces physical results.

\subsection{Two Scatters: Atmosphere, then Surface (_10)}

We also explore the behavior of the orange-rind planeparallel atmosphere in SRTC++ where photons experience two scatters. While SRTC ++ tracks the signal independently for any number of scatters, and where they occur, with elephant detectors, here we look at just the two-scatter case for ease of comparison to the semianalytical model. This case is quite a bit trickier for the analytical model in that we need to integrate over the upward hemisphere as seen from the surface, which encompasses all of the possible paths that the photon could take between its initial atmospheric scatter and its eventual surface scatter. We parameterize that hemisphere in terms of the zenith distance $\zeta$ and the azimuthal angle $\theta$ in the sky as seen from the surface scatter point. The hemispherical integral becomes one over $\zeta$ and $\theta$ such that

$$
\begin{aligned}
\frac{I_{-10}}{F}= & \pi \omega e^{-\tau \alpha(e)} \\
& \times \int_{0}^{\pi / 2} \int_{0}^{2 \pi} p(i, \zeta, \theta) \frac{\alpha(i)}{\alpha(i)-\alpha(\zeta)} \Phi_{s}(\zeta, e, \theta) d \theta d \zeta .
\end{aligned}
$$

where now both the phase function for the surface $\Phi_{s}$ and that for the atmosphere $p$ drive the final flux. We compute this 


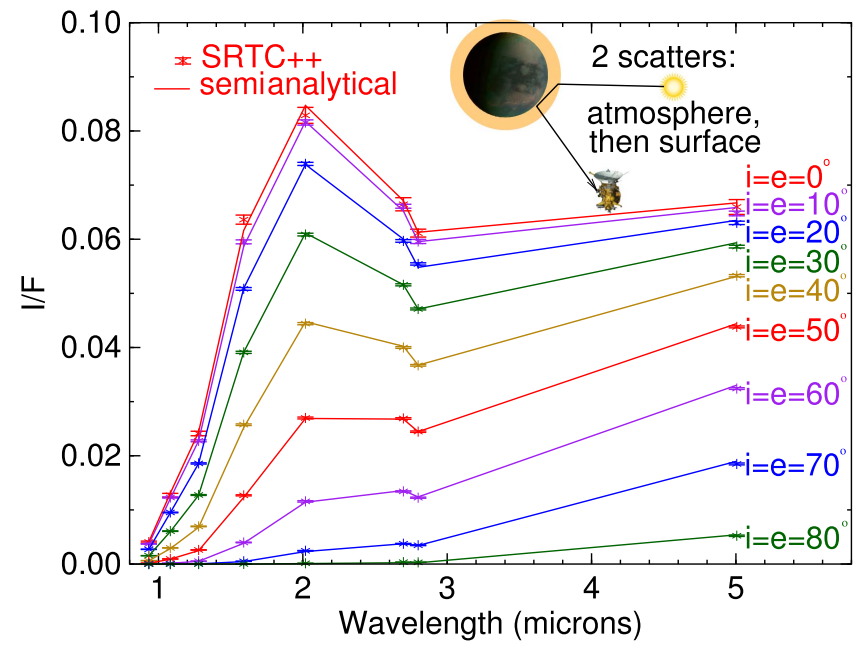

Figure 7. We plot here the results of double-scattering radiative transfer calculations for the $I / F$ as a function of wavelength for a Titan-like orange-rind atmosphere. In particular, here we only track those photons that first scatter off of the atmosphere, and then scatter off the surface to the detector. Because only a fraction of the total photons simulated experience this precise history, the $S$ / $\mathrm{N}$ in the SRTC++ data has decreased relative that in Figure 5. However the results agree well between the Monte Carlo SRTC++ and the semianalytical model from Equation (9).

nested integral numerically. If you try it yourself, take care to ensure that your inputs to the phase functions appropriately correspond to the angles between the incident and intermediate vector for the atmospheric phase function, and for the intermediate to emission vector for the surface phase function.

The result of that numerical integration of Equation (9) we show in Figure 7, along with the equivalent SRTC++ answer. The models track together across $80^{\circ}$ in incidence and emission. In fact, they track surprisingly well-at least partially as a result of the squat $30-\mathrm{km}$ atmospheric extent imposed on the SRTC++ calculations for the precise purpose of testing their comparison to the plane-parallel semianalytical model.

We show a spatial example of the results in Figure 8 as viewed from the detector with $90^{\circ}$ phase. The bright red near the terminator of the semianalytical model is bogus as the very high incidence angle near the terminator invalidates planeparallel results. The pixel-to-pixel noise in the SRTC++ image is real- the more photons in the simulation, though, the higher the signal-to-noise gets.

\subsection{Two Scatters: Surface, then Atmosphere (_01)}

The complement of Equation (9), photons bouncing first from the surface then from the atmosphere and to the detector, is given by

$$
\begin{aligned}
\frac{I_{-01}}{F}= & \pi \omega e^{-\tau \alpha(i)} \\
& \times \int_{0}^{\pi / 2} \int_{0}^{2 \pi} p(i, \zeta, \theta) \frac{\alpha(e)}{\alpha(e)-\alpha(\zeta)} \Phi_{s}(\zeta, e, \theta) d \theta d \zeta .
\end{aligned}
$$

The hemisphere of integration remains the sky from the surface point, but now the intermediate segment comes after that surface scatter instead of before it. Owing to the similarity between Equation (9) and Equation (10), the two equations yield the same answer regardless of the atmospheric phase function in the case
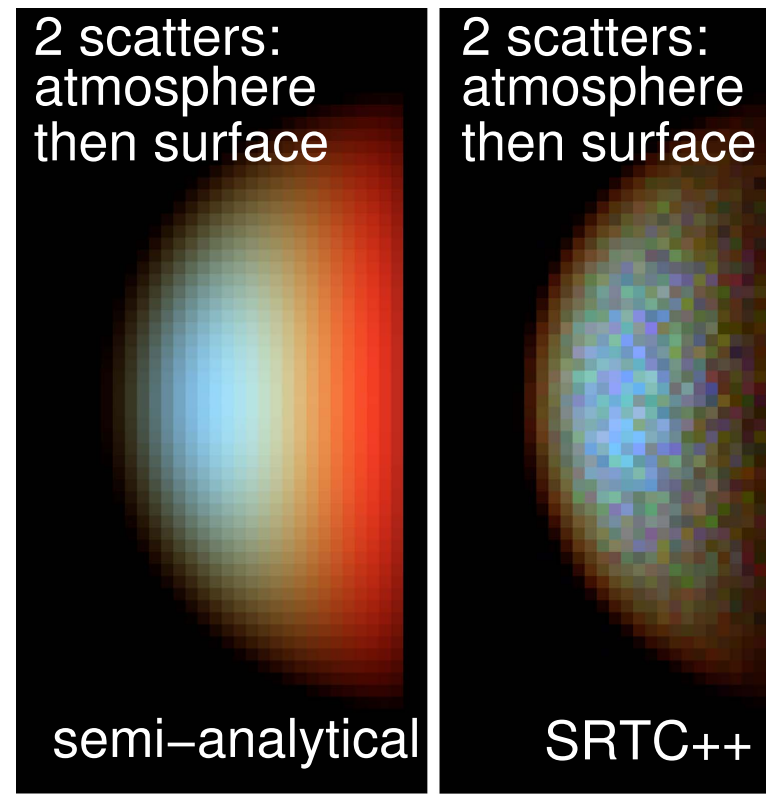

Figure 8. These images show the spatial results from the 10 calculations described in Section 3 for both the semianalytical model (left, from Equation (9)) and SRTC++ (right). The relatively low S/N evident in the pixel-to-pixel variation results from (1) selecting only that fraction of photons that experience the specific history _10 (i.e., ones that first bounce off of the atmosphere, then bounce off of the surface and arrive at the detector) and (2) using this many photons allowed us to sufficiently demonstrate that SRTC++ satisfactorily reproduces known plane-parallel results as shown in Figure 7. Running additional photons always improves the $\mathrm{S} / \mathrm{N}$, but takes progressively longer owing to the $\mathrm{S} / \mathrm{N}$ 's dependence on the square-root of the number of photons input. The colors map red to $5.0 \mu \mathrm{m}$, green to $2.0 \mu \mathrm{m}$, and blue to $1.3 \mu \mathrm{m}$. This image depicts a uniform sphere of albedo 1.0 illuminated from the left at $90^{\circ}$ phase angle. Hence the strong red signal in the semianalytical case comes from geometry near the terminator with high incidence angle $i-\mathrm{a}$ regime in which its plane-parallel assumptions break down.

where the surface phase function is Lambertian. The $I / F$ differs between the _10 and _01 cases under non-Lambertian surface phase functions (we see a difference when using an isotropic surface phase function, for example).

Given the assumed Lambertian surface scattering we see results in Figure 9 for the surface-then-atmosphere case are consistent with those from the atmosphere-then-surface case. The noise in the SRTC++ calculations is higher here, though, because of the highly forward-scattering nature of Titan's haze particles (from the Tomasko et al. (2008) phase function). Those fewer photons whose direction after their surface scatter brings them to within a few degrees of being pointed at the detector contribute more strongly to the detected intensity; hence the statistics of those relatively smaller numbers yields higher noise in Figure 7 than in Figure 9. Overall, the results of the SRTC++/semianalytical comparisons give us confidence in the SRTC++ calculations.

\subsection{Plane-Parallel Titan}

For a final validation, we compare SRTC ++ calculations to those of the existing Spherical Harmonic Discrete Ordinate Method plane-parallel radiative transfer model from Evans (2007) adapted for Titan by Hirtzig et al. (2013). We ran the Hirtzig et al. (2013) model without gaseous absorption and for endcases with surface albedo $A=0.0$ (all atmosphere) and $A=1.0$. For purposes of validation of $\mathrm{SRTC}++$, the Hirtzig et al. (2013) model run assumes a Tomasko et al. (2008) 


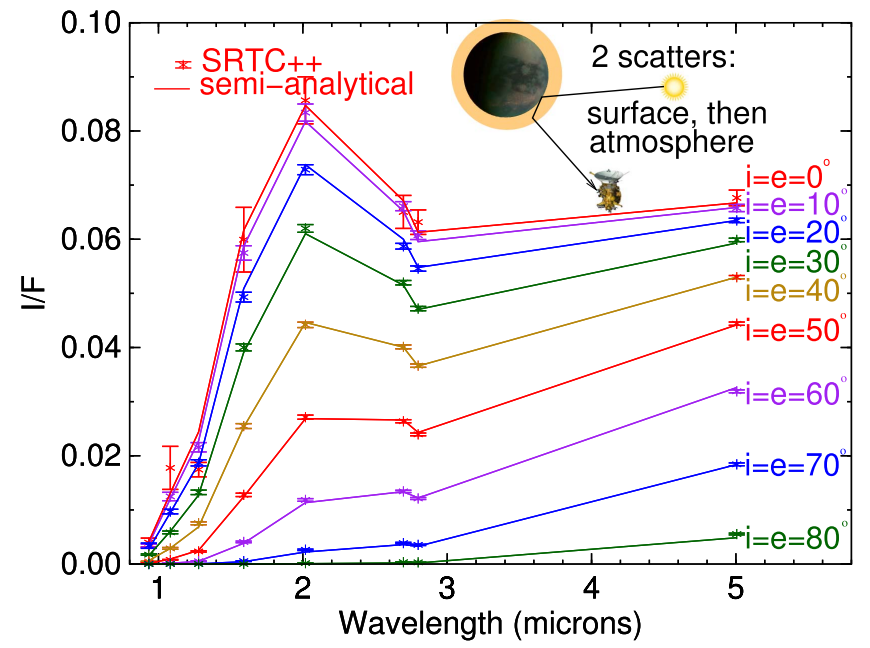

Figure 9. This figure represents our last comparison between SRTC++ and the semianalytical model. In this two-scatter, surface-then-atmosphere case, the lines show Equation (10) and the asterisks with error bars show the SRTC++ result. Although the semianalytical values match those from the atmospherethen-surface case (Figure 7), the higher SRTC++ noise here (i.e., lower S/N) results from Titan's highly forward-scattering haze particles' phase function (see the text).

atmospheric profile and haze phase functions, with interpolated single-scattering albedos between the high and low layer values within the middle atmospheric layer.

We then set up SRTC++ with an analogous atmosphere, but compressed vertically by a factor of 100 to more closely emulate the plane-parallel assumptions of the Hirtzig et al. (2013) model. We use two separate runs of 135,636,528 photons each, one for $A=0$ and one for $A=1$, with the photon generator emitting photons concentrated on areas with the appropriate geometry. We ran each run on a separate computer over a 3-day weekend.

Figure 10 shows the resulting intercomparisons. The Hirtzig et al. (2013) plane-parallel model and SRTC++ both agree from $i=e=0^{\circ}$ through $i=e=50^{\circ}$.

\section{Duration}

A drawback to Monte Carlo methods for 3D radiative transfer is that they tend to be slow. We have offset this weakness with parallelization and a fast implementation in $\mathrm{C}+$ + , but undoubtedly we can optimize further. In the meantime, we show an indication of how fast SRTC++ runs right now in Figures 11 and 12.

Figure 11 plots the computational speed in terms of throughput in photons per second as a function of the number of CPU cores that we use in the calculation. These runs executed on a dual 8 -core (16 total cores) $3.2 \mathrm{GHz}$ machine using the Titan model atmosphere from Section 3.7 with 57800 photons per run. As we designed the SRTC++ algorithm for each photon to calculate almost entirely independently from the others, the program is "embarrassingly parallel" in that we see only minor degradation in throughput per core even up to 16 cores executing simultaneously.

Atmospheric optical depth $\tau$ also significantly affects compute time. In Figure 12, we show the computation time for 57800 photons in uniform-extinction orange-rind atmospheres of differing optical depths. Computation times increase greater-than-linearly with optical depth, so while radiative transfer on Titan at $2 \mu \mathrm{m}$ where $\tau=1$ proceeds rapidly,

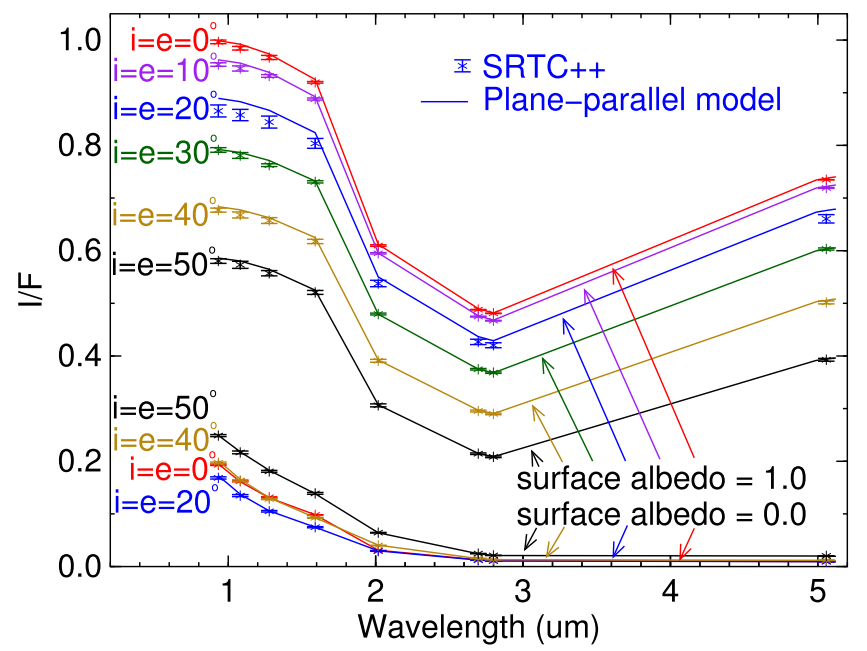

Figure 10. This plot compares SRTC++ results to those from the Hirtzig et al (2013) plane-parallel model for a Tomasko et al. (2008) Titan atmosphere with no gaseous absorption. The top set of points correspond to a model with surface albedo $A=1.0$, while the lower plots show results for a black $A=0$ surface.

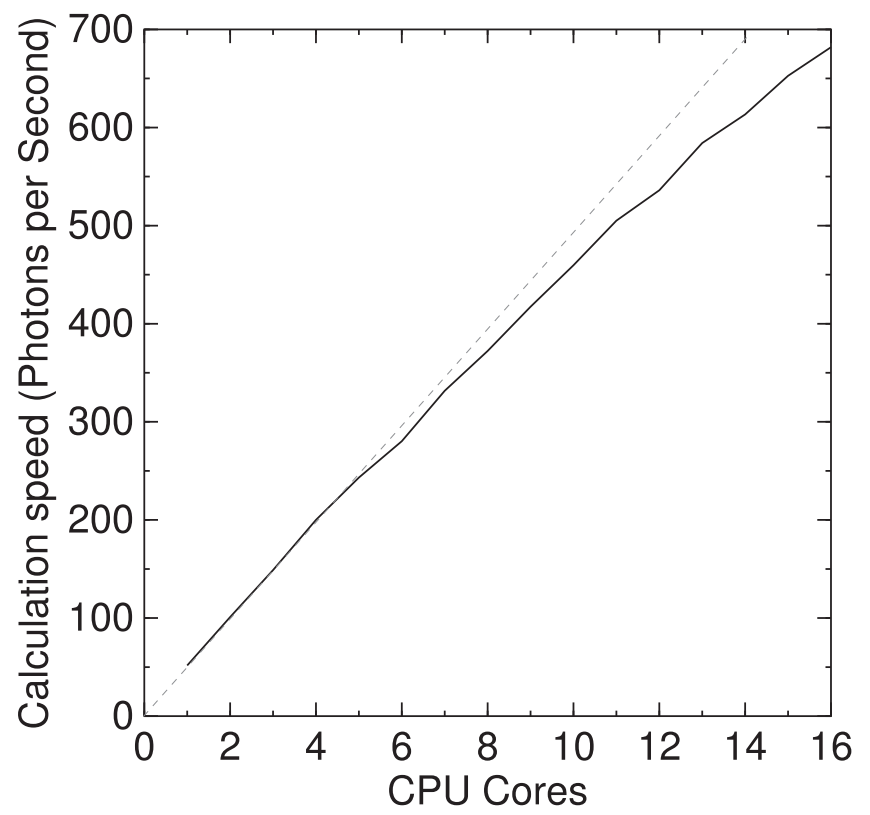

Figure 11. In this first of two benchmark graphs, we show SRTC ++ calculation speed as a function of the number of CPU cores used in the calculation. The throughput is nearly linear, with a small concave-down aspect resulting from the computation overhead of running in parallel. On the whole, the system scales well in this CPU regime. The gray dashed line provides a reference to guide the eye in assessing deviations from straight-line behavior.

calculations at visible wavelengths where $\tau \sim 10$ take considerably longer.

\section{Application}

We developed SRTC++ to complement existing planeparallel codes. Those models do a good job of modeling Titan near-IR reflectance spectra in geometries $i \lesssim 60^{\circ} e \lesssim 60^{\circ}$ where the plane-parallel approximation holds. SRTC ++ will model spatial problems, creating simulated images to characterize near-limb and near-terminator geometries, imaging resolution and surface nonuniformity, surface phase functions, specular reflections, and other problems. 


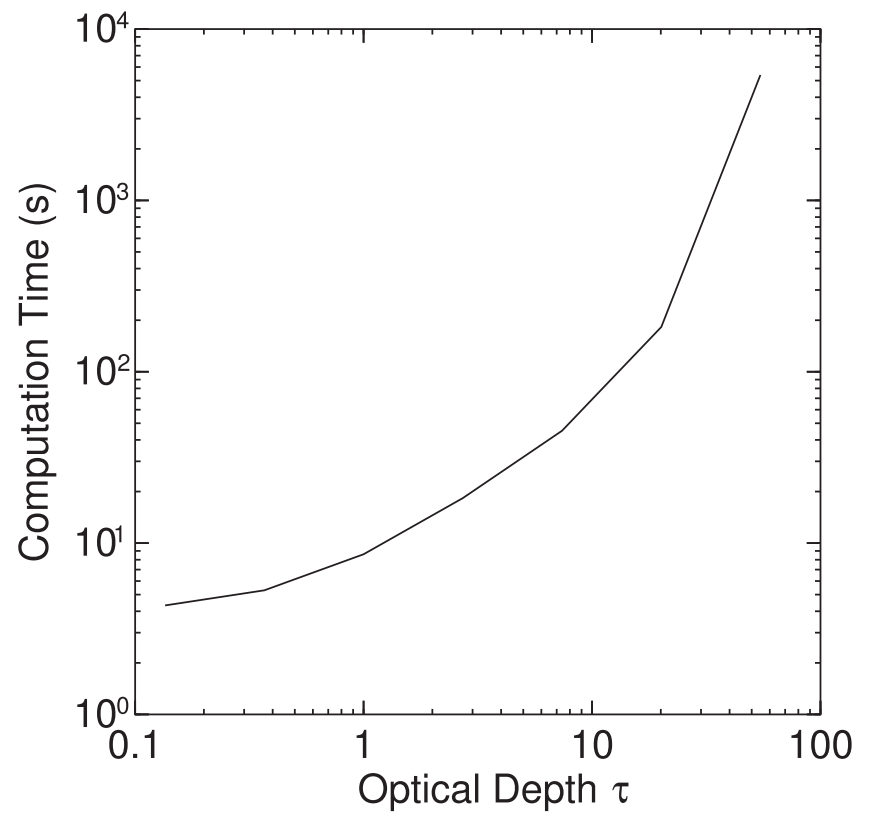

Figure 12. We plot here the total computation time for an uniform orange-rind atmosphere with different optical depths. Higher optical depths require much longer to complete; it follows that SRTC++ will be slow when simulating, for instance, radiative transfer through Titan's atmosphere at optical wavelengths.

To illustrate SRTC++'s capabilities, we use it to calculate emission phase functions for Titan, which we show in Figure 13. Complementary to our earlier figures, like Figure 7, which assumes specular geometry with an incidence angle equal to the emission angle, Figure 13 uses a fixed incidence angle of $60^{\circ}$ and varies only the emission angle. If you were a spacecraft starting right above the illuminated point on the 4PM afternoon equator, you could acquire an emission phase function by staring at that point as you moved around the moon. But you could do so in any direction. To show how that direction would affect your measurements, we show the emission phase function as if you were in a prograde equatorial orbit heading toward the terminator and looking back (dark green), as if you were in a retrograde equatorial orbit heading toward noon (red), and as if you were in a polar orbit (blue). The differences between these azimuths result from the backscattering (red) and forwardscattering (dark green) properties of Titan's atmospheric haze.

The SRTC++ algorithm as we describe it here represents an initial first-cut that can start to address useful problems. However, we plan many improvements and optimizations in the future as needed. For instance, right now SRTC ++ only accounts for gaseous absorption within the atmospheric singlescattering albedo parameter. As a future improvement, we intend to separately treat haze and gas opacities, using correlated- $k$ coefficients to simulate VIMS' rather coarse spectral resolution elements. SRTC ++ will probably never be the best choice for large problems that require high spectral resolution, however, like computing radiative atmospheric heating rates.

While at present SRTC++ includes a canned Tomasko et al. (2008) atmospheric model, Doose et al. (2016) since described improvements on the Tomasko et al. (2008) atmospheric model. It follows that while we will maintain the present model capabilities, future applications of SRTC ++ will preferentially assume the Doose et al. (2016) Titan haze scattering properties.
The surface phase functions included in SRTC++ now (just isotropic and Lambertian) are azimuthally symmetric. However, Buratti et al. (2006) showed a long time ago that Titan's surface does not obey a Lambertian law but rather shows significant backscattering properties. Buratti et al. (2006) used a Henyey-Greenstein surface phase function. But with better knowledge of diffuse atmospheric illumination from SRTC++, along with an additional 12 years of VIMS data, we hope to infer more detailed surface properties on Titan's various terrains from their phase functions using $\mathrm{SRTC}++$.

Specular reflections from Titan's lakes and seas (Stephan et al. 2010; Barnes et al. 2011; Soderblom et al. 2012) will prove a separate challenge. Purely specular surfaces will require separate calculations of two different paths to each detector through the specular point. Specular reflections from a roughened surface (like the wavy Punga mare described in Barnes et al. 2014), however, can be modeled with the present construction via particular non-azimuthally symmetric phase functions.

Because of its inherently 3D structure, SRTC++ can also simulate atmosphere-only phenomena. In particular, limb observations of atmospheric haze, stellar and/or solar occultations, and Titan's winter south polar cloud (West et al. 2016) would be amenable to analysis using SRTC ++ .

Thinking bigger-picture, SRTC ++ can also be applied to the photometric and spectroscopic properties of exoplanets. Direct detections of planets (e.g., Kalas et al. 2008) measure diskintegrated planetary properties; $\mathrm{SRTC}++$ could be used to accurately forward-compute expected photometric behavior of such planets as a function of phase as they orbit their parent star (Cahoy et al. 2010). SRTC++ might also be profitably applied to transit spectroscopy of planets (Hubbard et al. 2001) particularly those with thick and/or extended Titan-like atmospheres (Checlair et al. 2016). The high slant optical depths in such cases (Fortney 2005), potentially combined with east-west and equator-pole inhomogeneities (Fortney et al. 2010), lend themselves naturally to SRTC++'s explicit and accurate approach.

While not yet incorporated, the detector design allows for future intelligent coaddition of colorCCD's to maximize CPU time. For instance, after a single run, if the user desires a higher $\mathrm{S} / \mathrm{N}$, then results from a second run could be coadded with those of the first run. Similarly, separate instances of SRTC++ could be run on different computers, only to have their $\mathrm{CO}$ lor CCD results combined later to amalgamate separate computers as a greater cluster.

Right now, SRTC++ uses OpenMP for parallelization. OpenMP unlocks all of the cores on a single computer for use. A future improvement might be to use the alternate parallelization scheme MPI, which allows networks of computers to all contribute their CPU cores toward a particular problem. Such a change would enable SRTC++ to run on large supercomputers that do not use shared memory as well.

The compilation process might be facilitated by use of a photon generator more sophisticated than that of our photongenerator_square. The raster pattern performs much better than an entirely random photongenerator in that its noise drops as $N^{-1}$ as opposed to $N^{-\frac{1}{2}}$ (Press et al. 2007, page 404). However, it requires that the user decide in advance how many photons to use. It would be straightforward to instead implement a new photongenerator to make use of the Sobol' (1967) distribution. The Sobol' sequence is subrandom 

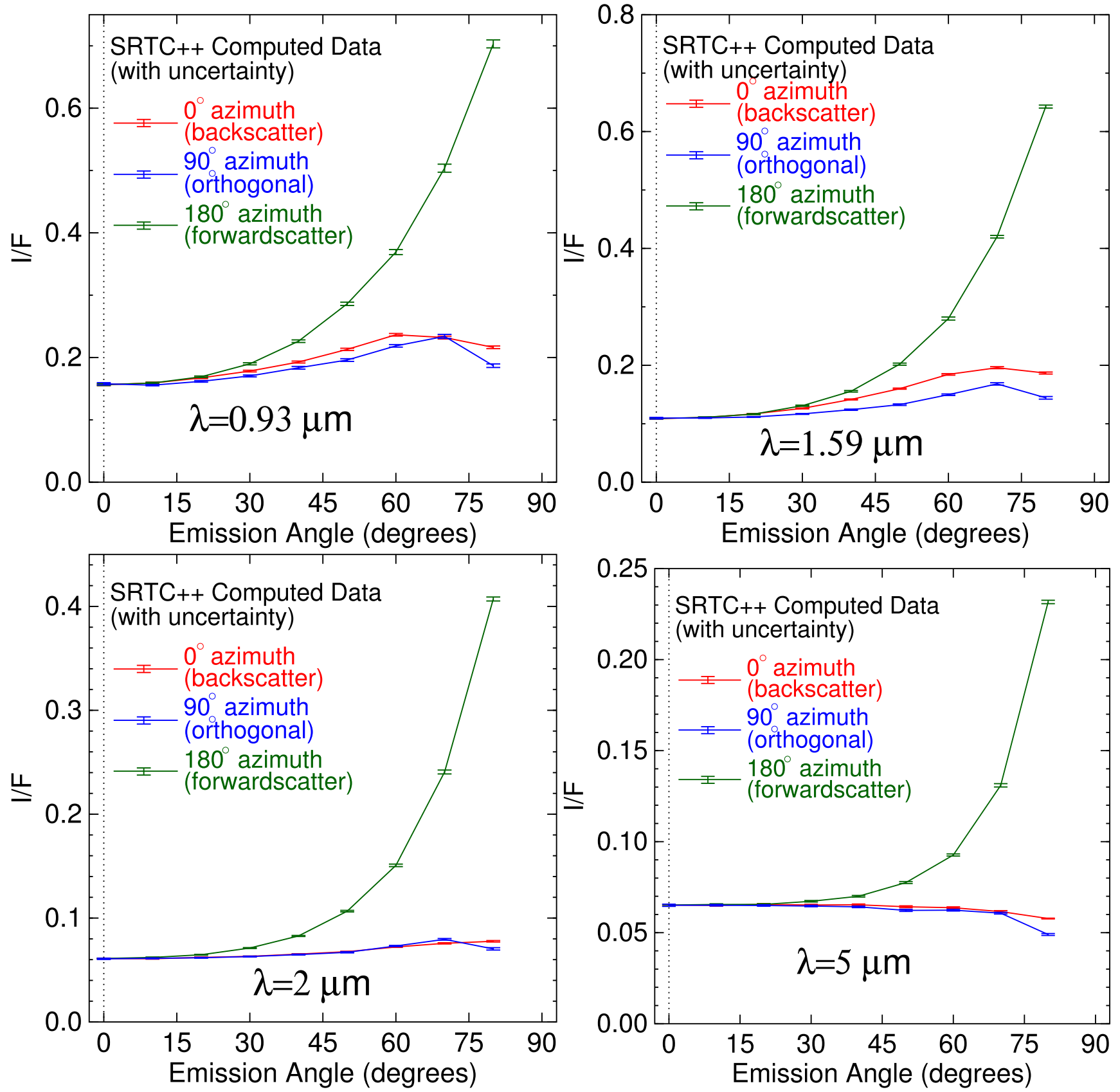

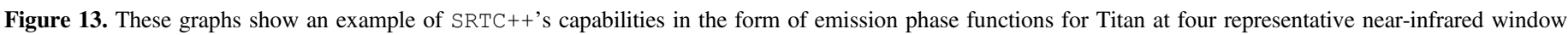

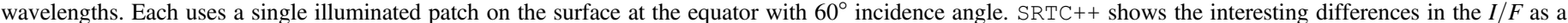

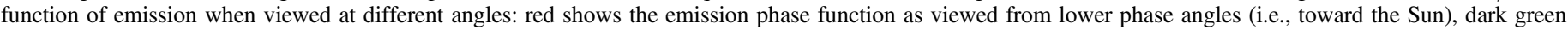

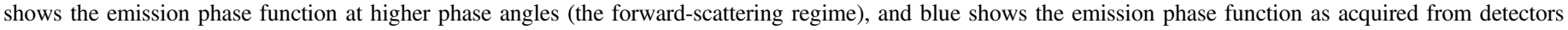
heading north from the illuminated patch (orthogonal to the Sun-Titan-Spacecraft plane).

an deterministic, but progressively fills in holes left in 2D space such that it could be cut off at an arbitrary point without introducing spatial irregularities (see Press et al. 2007, Figure 7.8.1).

Further speedup may be possible using an exact precomputation of the single-scattering component. Because most of the signal in a SRTC ++ output comes from singly scattered photons (either _ 0 or $\_1$ ), most of the noise comes from that component, too. If the single-scattered components of an elephant detector were assigned as semianalytical values, then the net noise would depend only on the higher-order scattering components. The ultimate result of such a modification would be higher precision results with fewer input photons.

As written, SRTC ++ depends on other packages and thus requires specific effort to install on operating system architectures other than that on which we wrote it (FreeBSD). It follows that we provide access to $\mathrm{SRTC}++$ via a whole-disk image of a working FreeBSD system that can be run from a thumb drive or as a virtual machine. You can find that disk image at the SRTC++ github repository located at https:// github.com/SRTCpp/Code where we also include a copy of the primary source code files (which are also accessible via DOI as doi: 10.5281/zenodo.1193815). 
The authors acknowledge the support of the NASA/ESA Cassini mission. J.W.B. acknowledges support from the NSF Astronomy \& Astrophysics (A\&A) Program grant \#1313427. S.M.M., E.F.Y., and J.M.S. acknowledge support from NSF grant \#1518226. Additional support was provided by Hubble program number HST-GO-12900.003-A from NASA through a grant from the Space Telescope Science Institute, which is operated by the Associate of Universities for Research in Astronomy, Incorporated, under NASA contract NAS5-26555. S.R. is partly supported by the Institut Universitaire de France. S.R. and T.C. also acknowledge financial support from the UnivEarthS LabEx program of Sorbonne Paris Cite (ANR-10LABX-0023 and ANR-11-IDEX-0005-02) and the French National Research Agency (ANR-APOSTIC-11-BS56-002 and ANR-12-BS05-001-3/EXO-DUNES). The authors acknowledge Johnathon Ahlers for insights into compiling SRTC++ under Linux. Thanks to reviewer Chris McKay and to editor Thomas Robitaille for constructive comments.

\section{ORCID iDs}

Jason W. Barnes (iD https://orcid.org/0000-0002-7755-3530

Brian K. Jackson (ib https://orcid.org/0000-0002-9495-9700

\section{References}

Ádámkovics, M., Mitchell, J. L., Hayes, A., et al. 2016, Icar, 270, 376 Baes, M., Davies, J. I., Dejonghe, H., et al. 2003, MNRAS, 343, 1081 Barnes, J. W., Brown, R. H., Soderblom, L., et al. 2007, Icar, 186, 242 Barnes, J. W., Brown, R. H., Turtle, E. P., et al. 2005, Sci, 310, 92 Barnes, J. W., Clark, R. N., Sotin, C., et al. 2013, ApJ, 777, 161

Barnes, J. W., Soderblom, J. M., Brown, R. H., et al. 2009, P\&SS, 57, 1950

Barnes, J. W., Soderblom, J. M., Brown, R. H., et al. 2011, Icar, 211, 722

Barnes, J. W., Sotin, C., Soderblom, J. M., et al. 2014, PlSci, 3, 3

Buratti, B. J., Sotin, C., Brown, R. H., et al. 2006, P\&SS, 54, 1498

Cahoy, K. L., Marley, M. S., \& Fortney, J. J. 2010, ApJ, 724, 189

Chandrasekhar, S. 1950, Radiative Transfer (Oxford: Clarendon Press)

Checlair, J., McKay, C. P., \& Imanaka, H. 2016, P\&SS, 129, 1

Coustenis, A., Lellouch, E., Maillard, J. P., \& McKay, C. P. 1995, Icar, 118, 87 Dlugach, J. M., \& Yanovitskij, E. G. 1974, Icar, 22, 66

Doose, L. R., Karkoschka, E., Tomasko, M. G., \& Anderson, C. M. 2016, Icar, 270,355

Dupree, S. A., \& Fraley, S. K. 2012, A Monte Carlo Primer: A Practical Approach to Radiation Transport (Berlin: Springer)

Evans, K. F. 2007, JAtS, 64, 3854

Fortney, J. J. 2005, MNRAS, 364, 649
Fortney, J. J., Shabram, M., Showman, A. P., et al. 2010, ApJ, 709, 1396 Griffith, C. A. 1993, Natur, 364, 511

Griffith, C. A., Doose, L., Tomasko, M. G., Penteado, P. F., \& See, C. 2012a, Icar, 218, 975

Griffith, C. A., Lora, J. M., Turner, J., et al. 2012b, Natur, 486, 237

Griffith, C. A., Penteado, P., Baines, K., et al. 2005, Sci, 310, 474

Hayne, P. O., McCord, T. B., \& Sotin, C. 2014, Icar, 243, 158

Hirtzig, M., Bézard, B., Lellouch, E., et al. 2013, Icar, 226, 470

Hubbard, W. B., Fortney, J. J., Lunine, J. I., et al. 2001, ApJ, 560, 413

Jonsson, P. 2006, MNRAS, 372, 2

Kalas, P., Graham, J. R., Chiang, E., et al. 2008, Sci, 322, 1345

Maltagliati, L., Bézard, B., Vinatier, S., et al. 2015a, Icar, 248, 1

Maltagliati, L., Rodriguez, S., Sotin, C., et al. 2015b, in European Planetary Science Congress 10, EPSC2015-687

McKay, C. P., Pollack, J. B., \& Courtin, R. 1989, Icar, 80, 23

Porco, C. C., Baker, E., Barbara, J., et al. 2005, Natur, 434, 159

Press, W. H., Teukolsky, S. A., Vetterling, W. T., \& Flannery, B. P. 1992, Numerical Recipes in C. The Art of Scientific Computing (Cambridge: Cambridge Univ. Press)

Press, W. H., Teukolsky, S. A., Vetterling, W. T., \& Flannery, B. P. 2007, Numerical Recipes: The Art of Scientific Computing (Cambridge: Cambridge Univ. Press)

Rages, K., \& Pollack, J. B. 1983, Icar, 55, 50

Rannou, P., McKay, C. P., \& Lorenz, R. D. 2003, P\&SS, 51, 963

Richardson, J., Lorenz, R. D., \& McEwen, A. 2004, Icar, 170, 113

Robitaille, T. P. 2011, A\&A, 536, A79

Rodriguez, S., Le Mouélic, S., Sotin, C., et al. 2006, P\&SS, 54, 1510

Smith, B. A., Soderblom, L., Beebe, R. F., et al. 1981, Sci, 212, 163

Smith, F. L., \& Smith, C. 1972, JGR, 77, 3592

Smith, P. H., Lemmon, M. T., Lorenz, R. D., et al. 1996, Icar, 119, 336

Sobol', I. M. 1967, ZVMMF, 7, 784

Soderblom, J. M., Barnes, J. W., Soderblom, L. A., et al. 2012, Icar, 220, 744

Soderblom, L. A., Kirk, R. L., Lunine, J. I., et al. 2007, P\&SS, 55, 2025

Solomonidou, A., Coustenis, A., Hirtzig, M., et al. 2016, Icar, 270, 85

Solomonidou, A., Hirtzig, M., Coustenis, A., et al. 2014, JGRE, 119, 1729

Squyres, S. W., McKay, C. P., \& Reynolds, R. T. 1985, JGR, 90, 12

Stephan, K., Jaumann, R., Brown, R. H., et al. 2010, GeoRL, 37, L7104

Thomas, G. E., \& Stamnes, K. 2002, Radiative Transfer in the Atmosphere and Ocean (Cambridge: Cambridge Univ. Press), 546

Tomasko, M. G., Archinal, B., Becker, T., et al. 2005, Natur, 438, 765

Tomasko, M. G., Doose, L., Engel, S., et al. 2008, P\&SS, 56, 669

Tomasko, M. G., \& West, R. A. 2010, in Titan from Cassini-Huygens, ed. R. H. Brown, J.-P. Lebreton, \& J. H. Waite (Berlin: Springer), 297

Vixie, G., Barnes, J. W., Bow, J., et al. 2012, P\&SS, 60, 52

Vixie, G., Barnes, J. W., Jackson, B., et al. 2015, Icar, 257, 313

West, R. A., Del Genio, A. D., Barbara, J. M., et al. 2016, Icar, 270, 399

Wolf, S. 2003, CoPhC, 150, 99

Xu, F., West, R. A., \& Davis, A. B. 2013, JQSRT, 117, 59

Young, E. F., Rannou, P., McKay, C. P., Griffith, C. A., \& Noll, K. 2002, AJ, 123,3473

Yusef-Zadeh, F., Morris, M., \& White, R. L. 1984, ApJ, 278, 186 